\title{
Relation Between ILAE Hippocampal Sclerosis Classification and Clinical Findings in Temporal Lobe Epilepsy
}

\author{
Ozan HASIMOGLU 1 , Ozan BARUT ${ }^{2}$, Meliha Ozge KAPAR ${ }^{3}$, Omer Batu HERGUNSEL ${ }^{4}$, Demet KINAY ${ }^{5}$, \\ Gunay $\mathrm{GUL}^{6}$, Ayten Ceyhan DIRICAN ${ }^{6}$, Bilge BILGIC${ }^{7}$, Bekir TUGCU²
}

\author{
${ }^{1}$ Kilis State Hospital, Department of Neurosurgery, Kilis, Turkey \\ ${ }^{2}$ University of Health Sciences Basaksehir Cam and Sakura City Hospital, Department of Neurosurgery, Istanbul, Turkey \\ ${ }^{3}$ Erzurum Regional Education and Research Hospital, Department of Pathology, Erzurum, Turkey \\ ${ }^{4}$ Koc University Hospital, Department of Neurosurgery, Istanbul, Turkey \\ ${ }^{5}$ University of Health Sciences Prof. Dr. Cemil Tascioglu City Hospital, Department of Neurology, Istanbul, Turkey \\ ${ }^{6}$ University of Health Sciences Prof. Dr. Mazhar Osman Bakirkoy Psychiatry and Neurological Diseases Research and Training Hospital, \\ Department of Neurology, Istanbul, Turkey \\ ${ }^{7}$ istanbul Faculty of Medicine, Department of Pathology, Istanbul, Turkey
}

Corresponding author: Ozan HASIMOGLU ozanhasim@hotmail.com

\section{ABSTRACT}

AIM: To investigate the relationship between clinical features and prognosis of the hippocampal sclerosis (HS) cases and International League Against Epilepsy (ILAE) histopathology classification.

MATERIAL and METHODS: A hundred patients with refractory epilepsy who were operated with the diagnosis of the Mesial Temporal Lobe Epilepsy were included in the study. Socio-demographic characteristics, clinical and family histories, post-operative ILAE and Engel epilepsy scores and diagnostic tests were recorded. At the same time, all of the pathological specimens were classified according to the new semi-quantitative ILAE classification. A significant statistical relationship was investigated between clinical data and HS-ILAE groups.

RESULTS: There were 36 male 64 female patients. The mean follow-up period was 6.5 years. $75 \%$ of the cases were HS-ILAE type 1, 19\% HS-ILAE type 2, 6\% were unidentifiable. FCD3A was detected in 3 patients. The HS-ILAE Type 2 ratio was high on the rightsided cases. In addition, HS-ILAE Type 1 ratio was high in patients with early seizure onset and long duration of epilepsy. There was no significant relationship between long-term ILAE and Engel epilepsy outcome scores and HS-ILAE types.

CONCLUSION: Resection of mesiotemporal structures in hippocampal sclerosis provides seizure control in at least two-thirds of cases. Histopathological findings may help us understand the epileptogenicity-prognosis of HS. The relationship between ILAE histopathology classification and clinical factors will become more obvious in the future. According to our study, there was a relationship between onset age of epilepsy, epilepsy duration, lesion side and HS-ILAE types. The reinforcement of these relationships with larger series will benefit clinicians.

KEYWORDS: Hippocampal Sclerosis, Epilepsy, Temporal Lobectomy, ILAE Classification

ABBREVIATIONS: CA: Cornu amonis, DNET: Disembryoplastic neuroepithelial tumor, EEG: Electroencephalography, FCD: Focal cortical dysplasia, HS-ILAE: ILAE histopathological hippocampal sclerosis classification, HS: Hippocampal sclerosis, ILAE: International league against epilepsy, IPI: Initial precipitating insults, MRI: Magnetic resonance Imaging, MTLE: Mesial temporal lobe epilepsy, MTS: Mesial temporal sclerosis, NPT: Neuropsychological test, PET-CT: Positron emission tomography-computed tomography

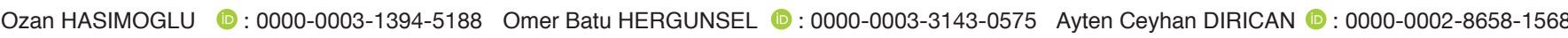
Ozan BARUT (1): 0000-0001-6572-9589 Demet KINAY Meliha Ozge KAPAR (10): 0000-0001-6751-758X Gunay GUL 


\section{INTRODUCTION}

E pilepsy is a very common neurological disease that has a prevalence of $0.5-1 \%$ according to the World Health Organization, and is detected in approximately 50 million people. Temporal lobe epilepsies are substantial in the etiology of drug resistant epilepsy. Approximately $22 \%$ of all seizures, and $60 \%$ of partial seizures are caused by temporal lobes (15). Although the pathologies of this region are very varied, Hippocampal Sclerosis (HS) or Mesial Temporal Sclerosis (MTS) are the most common ones with unique characteristics. HS is considered a prototype for drugresistant mesial temporal lobe epilepsies.

Hippocampal sclerosis has its own semiology and clinical course. After an effective respective surgery, full seizure control is achieved at a rate of $60-90 \%(7)$. HS is characterized with histopathological neuron loss and gliosis. The International League Against Epilepsy (ILAE) subcommittee presented a histopathological hippocampal sclerosis classification in 2013 (2). According to this classification, HS is histopathologically divided into three basic types, and three HS-related subtypes were also added, which are HS-ILAE Type 1 (typical), HS-ILAE Type 2-3 (atypical), No-HS (reactive gliosis only), Fragmented-HS (not excised en bloc), and FCD 3A (detection of focal cortical dysplasia with HS). Basically, the classification is mace according to anatomically sub-segments of the hippocampus; Cornu Amonis (CA1-4), Dentat Gyrus, fimbria, subiculum, parasubiculum, and entorhinal cortex based on degrees of cell loss.

The number of studies comparing ILAE classification and clinical findings and outcomes are limited. We aimed to compare the clinical, demographic and post-operative characteristics of 100 patients we operated due to HS with HS-ILAE Types.

\section{MATERIAL and METHODS}

\section{Study population}

This study was approved by the local ethic committee of the University of Health Sciences (Date: 06.03.2018; number: 149).

The study population was selected from among 125 patients diagnosed with drug resistant MTLE (Mesial Temporal Lobe Epilepsy) and treated surgically between 2009 and 2019. A total of 100 patients who met the inclusion criteria were included in the study (Table I). Preoperative clinical evaluations of the cases, the ages, genders, seizure start times, presence of first triggering event, anti-epileptics used, MRI, PET-CT, Video EEG, Neuropsychological Test Batteries were recorded. All patients underwent Anteromedial temporal lobectomy (Corticoamigdalohippocampectomy). The structures were excised by a single surgeon with microsurgery as en-bloc. The patients were re-evaluated face-to-face or with teleconference in 2019, and whether they had seizures, Engel and ILAE epilepsy scores were recorded $(5,19)$. The age, gender, epilepsy duration, epilepsy initial age, lesion side, MRI, Video-EEG, NPT (Neuropsychological Test), and PET-CT Lateralization, presence of the initial precipitating insults (IPI) (Febrile Convulsion, Neuro-infection, Trauma), history of epilepsy in first-degree relatives and dual pathologies of the patients were listed. The Engel and ILAE epilepsy scores of the patients in the $6^{\text {th }}$ month after the surgery and at last follow-up were also recorded. The epilepsy scores for 2019 were evaluated both alone and by being separating into good and poor prognosis groups.

\section{Preparation of Tissues}

The paraffin blocks of the tissues were taken from the archives for re-examination, and appropriate tissues were identified for immunohistochemical examination. A section was prepared for HE staining from the paraffin blocks to examine. In addition, 3-5 $\mu \mathrm{m}$ thick parts were prepared to be a section according to the number planned for immunohistochemical examination. The sections that would be used in the immunohistochemistry method were taken to positively charged slides. The prepared slides were dried and fixed in $56^{\circ} \mathrm{C}$ incubator overnight.

\section{Measurement Method}

Immunohistochemical procedures were performed on 100 HS cases as previously stated $(1,2)$. Neun, Neurofilament, and ps6 were applied to all cases that were considered to be FCD. Olig-2, IDH-1, CD34, and Neurofilament were studied to support the diagnosis in tumor cases. A ps6 was studied for a tumor case. Luxol Fast Blue was applied for hippocampal sclerosis evaluation. Neuronal loss zones were detected under 40x magnification.

\section{ILAE Classification Method}

The classification was carried out as stated in the ILAE commission report (2). In all CA1-4 hippocampal segments, common cell loss, and gliosis HS-ILAE Type 1, CA1 dominant cell loss HS-ILAE Type 2, CA4 dominant cell loss were considered as HS-ILAE Type 3 . In case detecting Focal Cortical Dysplasia coexistence, the classification was made

Table I: Inclusion and Exclusion Criteria

\begin{tabular}{|c|c|}
\hline Inclusion Criteria & Exclusion Criteria \\
\hline 1- Operated in $2009-2019$ by a single surgeon. & 1- The suspected diagnosis of hippocampal sclerosis \\
\hline $\begin{array}{l}\text { 2- Applying Video-EEG monitoring, T2 and T2 Flair MRI, PET-CT, } \\
\text { Neuropsychological Test Batteries before the operation. }\end{array}$ & 2- Irregular follow-up \\
\hline 3- Diagnosing unilateral MTLE & \\
\hline 4- Having regular follow-up and data recording per 6 months & \\
\hline
\end{tabular}


as FCD-3A, and in case no other findings were found except for reactive gliosis, the classification was made as no-HS. The tissues that were not excised en-block during surgery were considered as fragmented-HS.

\section{Statistical Analysis}

The SPSS 24 for Mac was used for statistical analysis while the findings obtained in the study were evaluated.

The Fisher Exact Chi-Square test was used since the expected frequencies were not met in comparing qualitative data.

In variables where parametric assumptions were met in comparing more than two groups, the One-Way ANOVA and Post Hoc Bonferroni were used; in variables where the parametric assumptions were not met, the Kruskal-Wallis test was used, and to determine the source of the difference, the Bonferroni Corrected Dunn Test was used.

The Bonferroni Correction was used where necessary, and the results of all other analyses except this correction were evaluated significant at $\mathrm{p}<0.05$ and $\mathrm{p}<0.01$.

\section{RESULTS}

A total of 100 patients were included in the study, and 64 of them were women, and 36 were male. The mean age was 30 years [11-54]. The mean follow-up period of the patients was 6.5 years [1-13]. A total of $46(46 \%)$ of the patients who participated in the study had lesions on the left, and 54 (54\%) on the right side.

The MRI revealed hippocampal sclerosis signs in the left side in 46 patients, and on the right side in 52, while 1 patient had bilateral hippocampal sclerosis. One patient had no MRI findings. Other tests are shown in Table II.

The MRI, Video-EEG, NPT findings of 65 (65\%) patients showed epileptogenic focus in the same mesial temporal side. We considered this a triple accordance. A total of 30 of these (30\%) were on the left, and 35 (35\%) were on the right.

When the characteristics that might be the IPI in patients' past, there were $47(47 \%)$ patients with a history of early Febrile convulsion, 32 (32\%) a history of trauma, $5(5 \%)$ had central nervous system infection, and 16 (16\%) had a history of epilepsy in first-degree relatives (Table II).

Dual pathology was detected in $10(10 \%)$ of the patients in pathological evaluations, and $3(3 \%)$ of these were reported as Focal Cortical Dysplasia (FCD3A), 2 (2\%) as Disembryoplastic Neuroepithelial Tumor (DNET), 4 (4\%) as Ganglioglioma, and 1 (1\%) Grade 2 Astrocytoma. All patients who were identified as FCD 3A were evaluated as ILAE Type 1.

According to Engel Epilepsy Classification in the follow-ups in the first sixth month after the surgeries, the patient distribution was; Engel 1A: 95, Engel 1B: 1, Engel 1D: 1, Engel 2B:1, Engel 3A: 1, Engel 4B:1. According to the scoring of ILAE epilepsy for the first six months, the number of patients was ILAE 1: 95, ILAE 3: 3, ILAE 4: 1, ILAE 5: 1.

In the final follow-ups (the average follow-up period was 6.5 years) for 2019, the same results were; Engel 1A: 76, Engel 1B: 4, Engel 1C: 3, Engel 1D: 8, Engel 2A: 5, Engel 2B:1, Engel 3A: 2, Engel 4B:1 and ILAE 1: 81, ILAE 3: 16, ILAE 4: 2, ILAE 5: 1 (Figure 1).

Table II: Demographic Features $(\mathrm{n}=100)$

\begin{tabular}{ll}
\hline Characteristic & $\mathbf{n}(\%)$, Means, \pm SD, (min-max) \\
\hline Gender (Female/Male) & $64(64) / 36(36)$ \\
\hline Mean age at surgery (years) & $29.99 \pm 8.95(11-54)$ \\
\hline Epilepsy duration (years) & $16.97 \pm 8.74(2-45)$ \\
\hline First seizure age (years) & $13.02 \pm 8.961-38$ \\
\hline 24h-VEEG R/L/B & $53(53) / 46(46) / 1(1)$ \\
\hline MRI R/L/B/N & $52(52) / 46(46) / 1(1) / 1(1)$ \\
\hline NPT R/L/B/N/Menthal Retardation & $41(41) / 34(34) / 18(18) / 5(5) / 2(2)$ \\
\hline PET-CT R/L/N & $19(19) / 13(13) / 2(2)$ \\
\hline Side of operation R/L & $54(54) / 46(46)$ \\
\hline Febrile seizure history & $47(47)$ \\
\hline Trauma history & $32(32)$ \\
\hline Infection history & $5(5)$ \\
\hline Family History & $16(16)$ \\
\hline
\end{tabular}

SD: Standard deviation, VEEG: Video EEG, NPT: Neuropsychological test batteries, MRI: Magnetic resonance imaging, PET-CT: Positron emission tomography-computed tomography. R: Right, L: Left, B: Bilateral, N: normal, n: Samples. 


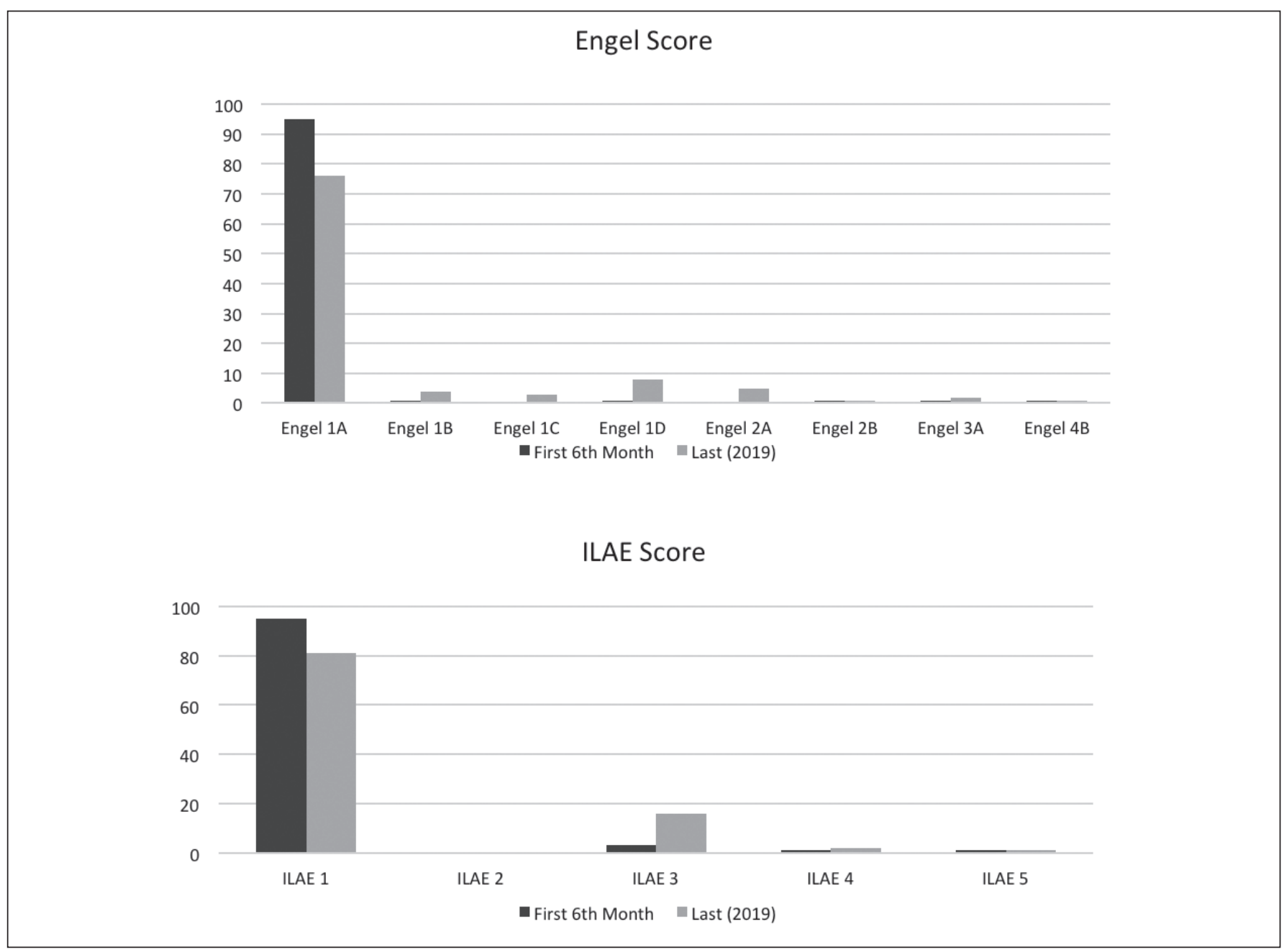

Figure 1: Follow-up of patients according to Engel and ILAE epilepsy classifications.

According to ILAE Pathology Classification, 75 (75\%) patients were Type $1 \mathrm{ILAE}$, and the number of patients with Type 2 was 19 (19\%) Six (6\%) patients were evaluated as unidentifiable. The unidentifiable finding will be addressed in the discussion.

No statistically significant differences were detected between the groups in terms of age and gender variables.

A statistically significant difference was detected between ILAE pathology classification groups in terms of seizure age variable. The seizure starting date of those who were ILAE Type1 was lower at a statistically significant level than that of ILAE Type $2(p<0.05)$.

In terms of the duration of the disease, there was a significant difference between the groups $(p<0.05)$. The duration of the disease of ILAE Type 1 individuals was higher at a statistically significant level than that of ILAE Type 2 individuals $(p<0.05)$.

The ILAE Pathology Classification groups were compared in terms of clinical features of the background and variables related to the IPI, and no statistically significant differences were detected between the groups (Table II).
When the patients were compared according to ILAE Pathology Classification Types, a statistically significant difference was observed between the groups in terms of the side of the lesion variable $(p<0.05)$. In this respect, the rate of those who had lesions on the right side in the ILAE Pathology Classification Type 2 Group was higher than Type $1(z=-2.31, p<0.05)$ and the Unidentifiable ones $(z=-2.08, p<0.05)$.

There were no associations between the results based on other diagnostic tests and the ILAE HS histopathological types. When the patients were compared according to ILAE Pathology Classification types, there were no statistically significant differences between the groups in terms of final follow-up Engel scoring prognosis variables ( $p>0.05$ ). This group was evaluated firstly according to good and then according to poor prognosis groups one by one, and two additional groups were added to the test and were tested separately. In the first comparison, although the good prognosis group was identified as Engel 1, and the poor prognosis group was identified as Engel 2, 3, 4, Engel 1 and 2 were accepted to the good prognosis, and Engel 3, 4 groups were accepted to the poor prognosis in the second 
comparison. In the first group, the target was to separate a subgroup that did not create complete disability, which could be considered almost seizure-free, from others. In the second group; however, our target was to distinguish the group without seizures or severely reduced seizures that benefited from the surgery, separating the group from the cases that did not benefit from the surgery or increased the number of seizures, making a distinction in the form of good and poor outcomes. The subclasses A, B, C, D of Engel scoring were not included in the comparison.

When the patients were compared according to ILAE Pathology Classification types, no statistically significant differences were detected between the groups in terms of recent ILAE Epilepsy scoring prognosis variables $(p>0.05)$. In this group, two different subgroups were created, including the good prognosis group (ILAE 1-2), and the poor prognosis group (ILAE 3-4-5-6). The target was to distinguish patients who lived without seizures or had only auras from other patients who had seizures. No significant differences were detected in this group (Table III).

\section{- DISCUSSION}

The MTLE is the most classic type of drug-resistant epilepsy, and the most common histopathology among these is hippocampal sclerosis. The main questions are whether these cases will benefit from surgery and whether they will be better in functional terms. It is important that etiopathogenetic differences can be important, and based on this, the preoperative surgical outcome can be predicted.

The ILAE histopathology classification published in 2013 has been included in routine hippocampal sclerosis assessment by many neuro-pathologists (2). This quantitative method will help standardize the outcomes, and establish clinicalpathological relations. Since the classification was developed, clinicians started to investigate whether there was a link between clinical features and histopathology. If strong prognostic and clinical associations are detected in this way, surgical en bloc excision may become routine.

In our study, the histopathological examinations and surgeries were re-evaluated by one single pathologist and one single surgeon, which ensured that the whole group was quite homogeneous. When the histopathological types were evaluated, it was found that Type 1 was the most common group in the literature, and accounted for more than half of the cases (60-80\%). Type 2; however, was found as the second most common in the literature between $5 \%$ and $10 \%$. It is detected in less than $10 \%$ in Type 3 cases. The FCD Type $3 A$ cases in which focal cortical dysplasia were detected and also were reported in quite varying rates in the literature. In the series of Gales et al., it was reported to be observed at a rate of $79 \%$ (9).

When the rates in our study were evaluated, similar rates were found in the literature $(2,3)$. Type1 (75\%), Type 2 (19\%), FCD Type 3A (3\%) and Unidentified (6\%). Although it is interesting that there were no patients in the group classified as Type 3 in our study, we see that Type 3 patients are limited to only a few patients in some series.

The dual pathologies that accompany the pathological diagnosis of hippocampal sclerosis are also very important because of their effects on postoperative results. It can be considered that there is an effect of dual pathologies in patient groups with no adequate seizure control during the

Table III: Comparing with HS-ILAE Classification and Patients' Features

\begin{tabular}{|c|c|c|c|c|}
\hline $\mathrm{N}=100$ & HS-ILAE Type 1 & HS-ILAE Type 2 & HS-ILAE Unidentifiable & $\mathbf{p}$ \\
\hline Age $($ Mean \pm SD $)$ & $29.7 \pm 9.38$ & $30.10 \pm 7.08$ & $33.16 \pm 9.55$ & 0.664 \\
\hline \multicolumn{5}{|l|}{ Gender } \\
\hline Female n (\%) & $47(62.7)$ & $13(68.4)$ & $4(66.7)$ & \\
\hline Male n (\%) & $28(37.3)$ & $6(31.6)$ & $2(33.3)$ & 0.930 \\
\hline First Seizure Age (Mean \pm SD) & $11.80 \pm 8.47$ & $17.63 \pm 8.53$ & $13.67 \pm 12.73$ & $0.034^{*}$ \\
\hline Epilepsy Duration (Mean \pm SD) & $17.90 \pm 9.15$ & $12.47 \pm 5.59$ & $19.50 \pm 7.86$ & $0.039^{*}$ \\
\hline Febrile Seizure n (\%) & $40(53.3)$ & $6(31.6)$ & $1(16.7)$ & 0,07 \\
\hline Trauma n (\%) & $25(33.3)$ & $5(26.3)$ & $2(33.3)$ & 0.927 \\
\hline Infection n (\%) & $3(4)$ & $2(10.5)$ & $0(0)$ & 0.464 \\
\hline Family History n (\%) & $11(14.7)$ & $5(26.3)$ & $0(0)$ & 0.275 \\
\hline \multicolumn{5}{|l|}{ Operation Side } \\
\hline $\mathrm{R}$ & $38(50.7)$ & $4(21.1)$ & $4(66.7)$ & $0.034^{\star}$ \\
\hline L & $37(29.3)$ & $15(78.9)$ & $2(33.3)$ & \\
\hline
\end{tabular}

SD: Standard Deviation, R: Right, L: Left, $n$ : Samples. 
Table IV: Comparing with HS-ILAE Classification and Prognosis Scores $(n=100)$

\begin{tabular}{|c|c|c|c|c|c|c|}
\hline $\begin{array}{l}\text { Classification and } \\
\text { Prognosis Score }\end{array}$ & & $\begin{array}{l}\text { ILAE Type } 1 \\
(n=75)\end{array}$ & $\begin{array}{l}\text { ILAE Type } 2 \\
(n=19)\end{array}$ & $\begin{array}{l}\text { ILAE Non-type } \\
\qquad(n=6)\end{array}$ & & \\
\hline & & n (\%) & n (\%) & n (\%) & $\mathbf{c}^{2}$ & p \\
\hline \multirow[t]{4}{*}{ Engel Score } & 1 & $69(92.0)$ & $17(89.5)$ & $5(83.3)$ & 7.174 & 0.331 \\
\hline & 2 & $4(5.3)$ & $2(10.5)$ & $0(0.0)$ & & \\
\hline & 3 & $1(1.3)$ & $0(0.0)$ & $1(16.7)$ & & \\
\hline & 4 & $1(1.3)$ & $0(0.0)$ & $0(0.0)$ & & \\
\hline \multirow[t]{2}{*}{ Prognosis Group 1} & Good Prognosis (Engel Score 1) & $69(92.0)$ & $17(89.5)$ & 5 (83.3) & 1.320 & 0.534 \\
\hline & Poor Prognosis (Engel Score 2-3-4) & $6(8.0)$ & $2(10.5)$ & $1(16.7)$ & & \\
\hline \multirow[t]{2}{*}{ Prognosis Group 2} & Good Prognosis (Engel Score 1-2) & $73(97.3)$ & $19(100)$ & $5(83.3)$ & 3.496 & 0.256 \\
\hline & Poor Prognosis (Engel Score 3-4) & $2(2.7)$ & $0(0.0)$ & $1(16.7)$ & & \\
\hline \multirow[t]{4}{*}{ ILAE Epilepsy Score } & 1 & $60(80.0)$ & $16(84.2)$ & $5(83.3)$ & 6.606 & 0.383 \\
\hline & 3 & $13(17.3)$ & $3(15.8)$ & $0(0.0)$ & & \\
\hline & 4 & $1(1.3)$ & $0(0.0)$ & $1(16.7)$ & & \\
\hline & 5 & $1(1.3)$ & $0(0.0)$ & $0(0.0)$ & & \\
\hline \multirow[t]{2}{*}{$\begin{array}{l}\text { ILAE Epilepsy Score } \\
\text { Prognosis Group }\end{array}$} & Good Prognosis (ILAE Score 1-2) & $60(80.0)$ & $16(84.2)$ & $5(83.3)$ & 0.221 & 1.000 \\
\hline & Poor Prognosis (ILAE Score 3-6) & $15(20.0)$ & $3(15.8)$ & $1(16.7)$ & & \\
\hline
\end{tabular}

postoperative period. Therefore, as seen in our study; we think that instead of the excision of mesial temporal structures alone, the excision of mesial temporal structures with anterior temporal lobectomy would be more successful.

In the present study, the starting age of the disease was lower in the ILAE Type 1 group. Also, the duration of life with seizures before the surgical procedure was significantly longer with the HS-ILAE Type 1 group. This finding is also supported in a limited number of studies (4).

The patients with febrile convulsion, trauma and infection were evaluated in the background as the IPI in our study. In addition, patients with epilepsy in their family history were also evaluated in terms of having relations with ILAE pathology classification. This definition was identified as diseases like febrile seizures, meningitis, anoxia, trauma, birth trauma, intracerebral hematoma, which caused seizures that developed before the first 5 years of life, and it was argued that they could be in $40-60 \%$ of epileptic cases (11). Na et al. showed that patients with an IPI, which was described as a history of initial precipitating insults, had an association with HS-ILA Type 1 (12). It was reported that this condition is associated with the SCN1A gene mutation and the Granular Cell Proliferation occurring after the IPI. $(8,10)$ A significant difference was not detected between these groups in our study. Although it was found that the febrile convulsion history was more frequent in Type 1 patient groups, which was similar to the literature, this trend did not reach significance level in statistical terms. There were no clear correlations between epilepsy and family history.

A very interesting finding in our study was the correlation between the hemispheric side, in which hippocampal sclerosis was detected, and ILAE pathological classification. In approximately $79 \%$ of patients diagnosed with Type 2 based on the ILAE histopathological classification, the focus was on the right side, while in about $51 \%$ of the patients with Type 1 the focus was on the left side. This difference was statistically significant and was evaluated previously in the literature, and no significant results were found (4). Our study is the first one to detect a significant relation between the lesion side and the HS-ILAE subgroups. We could not explain this significance.

In the literature, it was reported that seizure-free life rates were between $75-85 \%$, and $40-50 \%$ in 10 -year followups in the first $1-2$ years $(6,13)$. It is undeniable that the amygdalohippocampectomy surgery in HS is more successful in drug-resistant temporal lobe epilepsy in appropriate cases than medical treatment. As seen in our series, full seizure control was achieved in $95 \%$ in the first 6-month follow-ups, and $76 \%$ of long-term follow-ups (mean 6.5 years) (Engel, $1 \mathrm{~A}$ ). When compared with the previous study conducted by Tugcu et al. with the same case group, the percentage of the patients 
without seizures increased for the first six months, which might be related to the increased experience of the surgical team (18). We assume that providing a higher seizure-free life than in the literature may be related with the choice of suitable patients, and the number of patients $(65 \%)$ who comply with lateralization in Video-EEG, NPT and MRG findings. However, it is noted in the literature that there is no homogeneity in the evaluations of patients in terms of lack of seizures after surgery. Some studies took the Engel classification as the basis, while others based their results on ILAE surgical results score, and in a small number of studies took into account both classifications.

We examined the relation between histopathological classification by doing both outcome classifications separately, even the sub-groupings in two different ways. In this way, it was possible to make comparisons with all previous studies. Similarly, although some publications referred to a full seizure-free life as a successful result, some other studies mentioned successful results as the presence of infrequent seizures that did not create disability, and comparisons were made considering this.

In our study, no clear relations were detected between ILAE histopathological classification groups and ILAE epilepsy and Engel Epilepsy Score groups. In addition, although the good and poor prognosis groups were separated to distinguish patients who have benefited significantly from surgery, i.e. those without seizures or with a reduced number of seizures, only with auras, no significant results were detected. Gales et al. conducted a study and did not detect a significant difference; however, they also showed that recurrent epilepsy decreased in the FCD Type 3 Group (9). However, there are studies in the literature showing that the best epilepsy control is provided in HS-ILAE Type 1 group $(4,12,14,17)$. Na et al. added that the success of the results was associated with the severity of hippocampal sclerosis. A similar correlation was also reported in the ILAE report, and it was mentioned that HSILAE Type 1 cases had better seizure-free life than Type 2 and $3(60-80 \%)$ in 2 -year follow-ups (2). In our study, the reason why no relation was detected between surgical results and histopathological subgroups might have been caused by HS not only being a hippocampal but also an extra hippocampal pathological condition (20). The ILAE report also mentioned that there were extra-hippocampal extensions in this area; and therefore, subgroups should be defined for more specific diagnoses. To differentiate the positive correlation from factors like surgical strategy and adequate resection, it was reported that studies with longer-term follow-ups should be conducted in wider case series (2).

This pathological classification also contains some question marks in it. A total of 6 patients were found to be unidentifiable in our study. The unidentifiable group here refers not to Fragmented-HS, but to a group that does not meet all these pathological classification criteria. The reason is that this group does not comply with the dominant neuron loss pattern in the identified subgroups. No classification proposals were made for these intermediate groups. The problem in classification stems partly from the fact that the neuron loss in ILAE classification is not quantitatively evaluated. In this respect,
Thom mentioned non-specific cases that could sometimes be included in two classes (16), which might require reworking on the classification. On the other hand, since the current classification identifies the majority of cases, the correlation between hippocampal sclerosis type and prognostic and background features can yield valuable results.

The number of cases in our study was large $(n=100)$, and the follow-up period was long (6.5 years on average), which are acceptable levels according to the literature (15). However, the fact that there were no cases matching the ILAE Type 3 pathological classification can be considered as the limitation of the study. In the future, it may be possible to establish the relation between the findings of the background and prognostic factors with the histopathological structure in case series where HS-ILAE Type 2 and 3 and other classification groups are more. Also, the fact that the number of FCD Type 3 cases did not allow any conclusions was another limitation. In addition, the evaluation of seizure follow-ups might have been a shortcoming. As a matter of fact, there might be a correlation between memory, cognition, and psychological state and HS-ILAE types.

\section{CONCLUSION}

Surgical resection of medial temporal structures for drugresistant mesial temporal lobe epilepsy is an effective intervention in appropriate cases, and is accompanied by a promising surgical result. The histopathological classification of the samples that were excised with this surgery will give us information about the course and history of the disease. More importantly, based on this information, we will have the chance to predict the surgery results of patients. As it can be seen in our study, the HS-ILAE Type 2 rate is significantly higher in early onset and long-term HS cases and in HSILAE Type 1 with a lesion on the right side. However, no clear associations were detected between prognostic parameters, initial triggering events, and socio-demographic variables and HS-ILAE types. As the number of cases increases, it will become more possible to clarify the clinical-pathological relations.

\section{REFERENCES}

1. Blümcke I, Coras R, Miyata H, Ozkara C: Defining cliniconeuropathological subtypes of mesial temporal lobe epilepsy with hippocampal sclerosis. Brain Pathol 22(3):402-411, 2012

2. Blümcke I, Thom M, Aronica $\mathrm{E}$, Armstrong $\mathrm{DD}$, Bartolomei $\mathrm{F}$, Bernasconi A, Bernasconi N, Bien CG, Cendes F, Coras R, Cross JH, Jacques TS, Kahane P, Mathern GW, Miyata $H$, Moshé SL, Oz B, Ozkara C, Perucca E, Sisodiya S, Wiebe $\mathrm{S}$, Spreafico R: International consensus classification of hippocampal sclerosis in temporal lobe epilepsy: A task force report from the ILAE commission on diagnostic methods. Epilepsia 54(7):1315-1329, 2013

3. Blümcke I, Thom M, Aronica E, Armstrong DD, Vinters HV, Palmini A, Jacques TS, Avanzini G, Barkovich AJ, Battaglia G, Becker A, Cepeda C, Cendes F, Colombo N, Crino P, Cross $\mathrm{JH}$, Delalande O, Dubeau F, Duncan J, Guerrini R, Kahane P, Mathern G, Najm I, Ozkara C, Raybaud C, Represa A, Roper 
SN, Salamon N, Schulze-Bonhage A, Tassi L, Vezzani A, Spreafico R: The clinicopathologic spectrum of focal cortical dysplasias: A consensus classification proposed by an ad hoc task force of the ILAE Diagnostic Methods Commission. Epilepsia 52(1):158-174, 2011

4. Deleo F, Garbelli R, Milesi G, Gozzo F, Bramerio M, Villani F, Cardinale F, Tringali G, Spreafico R, Tassi L: Short-and long-term surgical outcomes of temporal lobe epilepsy associated with hippocampal sclerosis: Relationships with neuropathology. Epilepsia 57(2):306-315, 2016

5. Engel $\mathrm{J} J \mathrm{~J}$ : Update on surgical treatment of the epilepsies summary of the second international palm desert conference on the surgical treatment of the epilepsies (1992). Neurology 43(8):1612-1617, 1993

6. Engel J Jr, Wiebe S, French J, Sperling M, Williamson P, Spencer D, Gumnit R, Zahn C, Westbrook E, Enos B: Practice parameter: Temporal lobe and localized neocortical resections for epilepsy. Epilepsia 44(6):741-751, 2003

7. Englot DJ, Chang EF: Rates and predictors of seizure freedom in resective epilepsy surgery: An update. Neurosurg Rev 37(3):389-404; discussion 404-405, 2014

8 Eriksson PS, Perfilieva E, Björk-Eriksson T, Alborn AM, Nordborg C, Peterson DA, Gage FH: Neurogenesis in the adult human hippocampus. Nat Med 4(11):1313-1317, 1998

9 Gales JM, Jehi L, Nowacki A, Prayson RA: The role of histopathologic subtype in the setting of hippocampal sclerosis-associated mesial temporal lobe epilepsy. Hum Pathol 63:79-88, 2017

10. Kasperaviciute D, Catarino CB, Matarin M, Leu C, Novy J, Tostevin A, Leal B, Hessel EVS, Hallmann K, Hildebrand MS, Dahl HHM, Ryten M, Trabzuni D, Ramasamy A, Alhusaini S, Doherty CP, Dorn T, Hansen J, Krämer G, Steinhoff BJ, Zumsteg D, Duncan S, Kälviäinen RK, Eriksson KJ, Kantanen AM, Pandolfo M, Gruber-Sedlmayr U, Schlachter K, Reinthaler EM, Stogmann E, Zimprich F, Théâtre E, Smith C, O’Brien TJ, Tan KM, Petrovski S, Robbiano A, Paravidino R, Zara F, Striano P, Sperling MR, Buono RJ, Hakonarson H, Chaves J, Costa PP, Silva BM, da Silva AM, de Graan PNE, Koeleman BPC, Becker A, Schoch S, von Lehe M, Reif PS, Rosenow F, Becker F, Weber Y, Lerche H, Rössler K, Buchfelder M, Hamer HM, Kobow K, Coras R, Blumcke I, Scheffer IE, Berkovic SF, Weale ME, UK Brain Expression Consortium; Delanty N, Depondt C, Cavalleri GL, Kunz WS, Sisodiya SM: Epilepsy, hippocampal sclerosis and febrile seizures linked by common genetic variation around SCN1A. Brain 136(Pt 10):3140-3150, 2013
11. Mathern GW, Pretorius JK, Babb TL: Influence of the type of initial precipitating injury and at what age it occurs on course and outcome in patients with temporal lobe seizures. J Neurosurg 82(2):220-227, 1995

12. Na M, Ge H, Shi C, Shen H, Wang Y, Pu S, Liu L, Wang H, Xie C, Zhu M, Wang J, Shi C, Lin Z: Long-term seizure outcome for international consensus classification of hippocampal sclerosis: A survival analysis. Seizure 25:141-146, 2015

13. Paglioli E, Palmini A, Paglioli E, da Costa JC, Portuguez M, Martinez JV, Calcagnotto ME, Hoefel JR, Raupp S, BarbosaCoutinho L: Survival analysis of the surgical outcome of temporal lobe epilepsy due to hippocampal sclerosis. Epilepsia 45(11):1383-1391, 2004

14. Sastri BVS, Arivazhagan A, Sinha S, Mahadevan A, Bharath RD, Saini J, Jamuna R, Kumar JK, Rao SL, Chandramouli BA, Shankar SK, Satishchandra P: Clinico-pathological factors influencing surgical outcome in drug resistant epilepsy secondary to mesial temporal sclerosis. J Neurol Sci 340(12):183-190, 2014

15. Téllez-Zenteno JF, Hernández-Ronquillo L: A review of the epidemiology of temporal lobe epilepsy. Epilepsy Research and Treatment 2012: 630853, 2011

16. Thom M: Review: Hippocampal sclerosis in epilepsy: a neuropathology review. Neuropathol Appl Neurobiol 40(5):520543,2014

17. Thom M, Liagkouras I, Elliot KJ, Martinian L, Harkness W, McEvoy A, Caboclo LO, Sisodiya SM: Reliability of patterns of hippocampal sclerosis as predictors of postsurgical outcome. Epilepsia 51(9):1801-1808, 2010

18. Tugcu B, Gungor A, Akpinar A, Kinay D, Kuscu DY, Gul G, Kayrak N, Keskinkilic C, Akdemir H, Emel E: Outcome of surgical treatment of hippocampal sclerosis from relatively new epilepsy surgery center. J Neurosurg Sci 60(2):159-168, 2016

19. Wieser HG, Blume WT, Fish D, Goldensohn E, Hufnagel A, King D, Sperling MR, Lüders $H$, Pedley TA, commission on neurosurgery of the International League Against Epilepsy (ILAE): ILAE commission report. Proposal for a new classification of outcome with respect to epileptic seizures following epilepsy surgery. Epilepsia 42(2):282-286, 2001

20. Wieser HG, ILAE commission on neurosurgery of epilepsy: ILAE commission report. Mesial temporal lobe epilepsy with hippocampal sclerosis. Epilepsia 45(6):695-714, 2004 